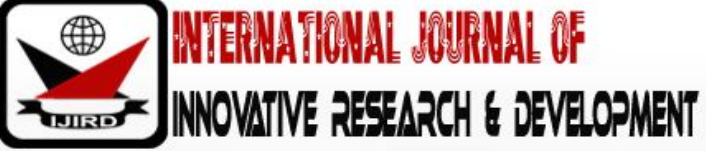

ISSN 2278 - 0211 (Online)

\section{Behaviour Change Communication in Health Promotion: Appropriate Practices and Promising Approaches}

\author{
\begin{tabular}{c} 
Samuel Ngigi \\
Lecturer, School of Journalism and Mass Communication, University of Nairobi, Kenya \\
Doreen Nekesa Busolo \\
Research Assistant, School of Journalism and Mass Communication, University of Nairobi, Kenya \\
\hline
\end{tabular}
}

\begin{abstract}
:
Behavior Change Communication may take different forms to appeal to individuals or groups to change behavior towards a specific health problem. The study focuses on how communication can be used as a potent tool for promoting positive health behavior among populations to prevent and control the spread of diseases and illnesses. The study also identifies models of communication that can be used to guide the development of strategies that foster protection, reduces risky behaviors, and encourage adoption and maintenance of positive behaviors. There are a wide variety of different communication channels ranging from basic face-to-face conversation, telecommunication channels like the telephone or e-mail, computational channels like the medical record and mass media channels like TV, radio, posters, brochures etc. It is important to know which channels can most effectively reach particular target populations. Campaigns attempt to influence adoption of recommended behaviors by influencing what people know and believe about their behavior, or influence actual or perceived social norms, or by changing actual skills and confidence in skills assumed to influence behavior. The study concludes that the effort to bring about positive behavior change in the society should involve all stakeholders.
\end{abstract}

Keywords: Behaviour change, behaviour change communication, models of communication

\section{Introduction}

World Health Organization (WHO), February 2018 reported that HIV continues to be a major global public health issue, having claimed more than 35 million lives. In 2016, 1.0 million people died from HIV-related causes globally. However, the key point to note is that the key populations often have legal and social issues related to their behaviors that increase vulnerability to HIV and reduce access to testing and treatment programs. Human behaviors have exposed individual to health risks thus affecting other aspects of life. For instance, a society that is invaded with health problems is at risk of being underdeveloped thus the need of behavior change.

WHO report (2009) on "Why health communication is important in public health"insists that many of the threats to global public health (through diseases and environmental calamities) are rooted in human behaviour. Effective communication is vital for an orchestrated response to a public health crisis (Haiders 2015). Communication as defined by Adler and Rodman (2003) refers to the process of human beings responding to the symbolic behavior of other persons. The three major components: communication is human, communication is a process and communication are symbolic are highlighted.

Communication serves an instrumental role that helps one acquire knowledge as well as also fulfilling ritualistic function, one that reflects humans as members of a social community (WHO 2009). Health communication attempts to persuade the affected group to engage in certain behavior through accessing information. Intervention measures to change behaviors in the affected group are an area of concern and must be fully supported by both the government and nongovernmental organizations.

Behavior Change Communication (BCC) is an essential component of development. However, society-wide change is slow. Majority of researchers of health communication insist on behavior change programs that aims at increasing knowledge, stimulating dialogue, promoting attitude change, reducing stigma, improve skills etc. Scutchfield and Keck (2003) insist that the success of communication is determined by a variety of factors that may include: how much access the target audience has to information, whether the target audience has acquired sufficient knowledge and skills to perform the behavior among others.

When changing behavior, the individual, community, or institution goes through a series of steps. Sometimes moving forward, sometimes moving backward and others may skip some steps. Even when individuals, communities, or institutions adopt new behaviors, they may at times revert to old behaviors, at least under certain circumstances.

To achieve this, WHO (2009) insist that in adopting the transmission view of communication, it is reasonable to think carefully about the channels through which intervention messages are disseminated, to whom the message is attributed, 
how audience members respond and the features of messages that have the greatest impact. The intervention message once is disseminated, it is received and processed through individual and social prisms that not only determine what people encounter but also the meaning that they derive from the communication depending upon factors at both the individual and the macro-social level.

Behavior change theories bare the roots of $\mathrm{BCC}$ and it is from these theories the comprehensive communication strategies and programs are developed. Communication theories according to Edberg (2015) are used to address among other things the process of encoding, sending, receiving and decoding messages. Giving example of the Health Belief Model (HBM) and Theory of Planned Behavior (TPB) the health behavior is constructed as a result of individual decision-making process or attitude that address health related issues.

Learning theory on the other hand explains the structural models and general social ecological theory. Learning theory is used to emphasize direct and vicarious learning through the interaction between behavior and consequences (Scutch field and Keck 2003). They further insisted that health communication and learning theory-based models provide divergent perspectives on planning and implementation of public health behavior change intervention.

Dissemination of message is important however the impact of the message to the recipient is what matters. To achieve this, there must be consideration of key components such as channel analysis. Bensley and Brookins-Fisher (2018) states that channel analysis helps to determine communication settings, channels and methods that are likely to appeal to the target group. This includes: where the priority group is most easily reached, how they receive information and their preferences.

\subsection{Background}

Behavior change is a very important aspect in health communication and isused as an intervention measure across a wide range of health problems. Chronic diseases, respiratory illnesses, HIV virus causing AIDS disease as well as zoonotic diseases can be prevented through health communication initiatives that can effectively promote positive behavior change.

Health communication is the study and practice of communicating promotional health information, such as in public health campaigns, health education, and between doctor and patient. The purpose of disseminating health information is to influence personal health choices by improving health literacy. In the same breath, the Centre for Disease Control and the National Cancer Institute has defined health communication as: "The study and use of communication strategies to inform and influence individual decisions that enhance health."

Behavior change communication (BCC) on the other hand is an interactive process of any intervention with individuals, communities and/ or societies (as integrated with an overall program) to develop communication strategies to promote positive behaviors which are appropriate to their settings. Strategic use of communication to promote positive health outcomes is based on proven theories and models of behavior change.

Milgrom (2015) insist that any successful communications program aimed at changing behavior must be grounded in theory. She further states health programs and companion communications campaigns should find components of at least one behavior-change theory. Most of the studies revealed that change of behavior differ across range of factors. Individual perceptions, beliefs or emotions affect decision-making towards behavior change. Interpersonal factors such as family influences like social support and relationship also affects individual decision-making. Community norms influence how people make decision regarding health issues. Structural factors such as the economic status of the country, political climate laws enforcement and policies also affects behavior change of an individual. Some behaviors are so tightly woven into social norms (e.g. smoking) that an ecological approach targeting the individual, as well as their community and broader environment, is critical. Milgrom2015).

Behavior change communication employs a systematic process that involves formative research and behavior analysis, communication planning, implementation, and monitoring and evaluation. In addition, the audiences are carefully segmented, messages and materials are pre-tested, and both mass media and interpersonal channels are used to achieve defined behavioral objectives. Self-affirmation strategy as identified by Martin and DiMatteo (2014), is used to increase receptiveness to potentially threatening health messages. The strategy allows reflection on individual's important values through engagement of open-minded and balanced appraisal.

\subsection{Definition of Basic BCC Models \& Channels}

Behavior Change Communication (BCC) has been defined by authors as an interactive process with communities to develop tailored messages and approaches using a variety of communication channels to develop positive behaviors; promote and sustain individual, community and societal behavior change; and maintain appropriate behaviors (FHI 2002). Behavior change communication (BCC) is the strategic use of communication to promote positive health outcomes, based on proven theories and models of behavior change (CDC 2018).BCC also employs a systematic process beginning with formative research and behavior analysis, followed by communication planning, implementation, and monitoring and evaluation. Audiences are carefully segmented, messages and materials are pre-tested, and both mass media and interpersonal channels are used to achieve defined behavioral objectives. Behavior change cannot happen overnight and so there are steps that can be followed to achieve the desired change as follows: pre-contemplative/ unaware, contemplative, preparing, action/ trying, maintaining and termination/ advocacy/transcendence.

On the other hand, there are factors that can prompt people to change their behavior. These factors include:physical stimuli based on a person's current physical state as well as fear of future pain discomfort or memory of past pain, rational stimuli based on knowledge of reasoning-if people have the facts they choose to do the right thing, emotional stimuli based on the intensity of feeling of fear, love or hope, skills based on the person's capacity to adopt and 
continue a new behaviour, family and personal networks based on the influence from family and peers, and social structures based on the impact of social economic legal and technological factors on the daily life of a person (FHI 2002). The study identified the key role of BCC as follows: to start or adopt a new behavior, to prevent the adoption of negative or harmful behavior, to change or modify an existing behavior, and to stop doing something damaging.

\subsubsection{BCC Models}

Behavioral models are designed to help researchers understand behavior and also seek to explain why people behave the way they do. Theories of change on the other hand seek to explain how behavior changes. There are different social psychological models that seek to explain human behaviors; however, there are common factors that were identified. Personal factors are intrinsic to the individual and this comprises of their level of knowledge or their belief in their ability to change their behavior and their habits (GCN and COI 2009).

Knowledge and awareness are personal factor that are rarely enough by themselves to bring about behavior change because other factors can override it. Providing information is the first step towards influencing behavior change rather than an end point.

Attitudes on the other hand are specific to particular behaviors. While attitudes can influence behavior, evidence now suggests that the link is not as strong as we might previously have thought (SNV 2016 report). The so-called Value Action Gap' describes those situations where a person holds values that are inconsistent with their behavior. For example, cognitive dissonance implies that a person holding two inconsistent views will feel a sense of internal conflict ('cognitive dissonance'), which will prompt them to change their views and so bring their perceptions into line. This has also been found to apply to inconsistencies between perceptions and behaviors.

Family Health International (FHI2002) insists that a good example of cognitive dissonance is evident among smokers. Most smokers some health professions (doctors) know that smoking causes lung cancer and other health problems, but they also want to live a long and healthy life. Smokers can seek to reduce this 'dissonance' either by giving up smoking or by finding ways to justify their habit. The more an individual repeat a particular behavior, the more automatic it becomes. As time passes and the behavior is undertaken more and more frequently.

Another personal factor which is self-efficacy agency describes an individual's sense that can carry out a particular action successfully and that action brings about the expected outcome. The important thing is the belief, not whether or not the individual is actually capable of achieving a particular goal. This determines the effort a person is prepared to put into changing their behavior and even whether they will attempt it at all (GCN and COI 2009).

Social factors are concerned with how individuals relate to each other and the influence of other people on their behavior. These factors are extremely important task during the formative stages of the strategic planning process. It helps to gain an understanding of the extent to which interpersonal influences are likely to be important for one or more target groups. Other people's values, attitudes, beliefs and behavior can have a strong social influence on our own behavior (UDSAID 2010).

Society has social norms that may vary among the communities. They are the group 'rules' that determine what acceptable behavior is and what is not. Social norms can have a huge influence on peoples' thoughts and behaviors. Social norms vary by group, so what the norm is for one group of may well be different from that adopted by another group living in different circumstances. Social proof is an aspect that is arrived at when people are unsure of how to act in social situations and often assume that others around them know more and look to them for pointers on how to behave (GCN and COI 2009).

Through communication, social norms are highlighted thus prompting people to act in accordance with them. Different approaches are used to facilitate this. For instance, peer-to-peer approaches such as online forums, positive testimonials from others who have adopted a particular behavior, targeting campaigns at respected, authoritative opinion leaders or recruiting them as 'ambassadors' for a brand or behavior and use of word of mouth.

Environmental factors are those factors that an individual have little control over. These include the facilities and activities found where and also the economy or technology. The physical conditions and environment in which people lives can help to understand why people behave in the way they do. The local environment motivates people to behave in a certain way especially if the local environment puts barriers in the way of the behavior people want to change or adopt Also the national or even international level can also have a huge influence on individual behavior (GCNand COI 2009).

As much as behavioral models are important they have limitations. Models are deliberately simplified to aid understanding, and 'do not account for all the complexities of behavior. Most models do not segment the target population however different factors are likely to carry different weight for different people. Models are usually based on data from a specific audience and/ or designed with a specific behavior in mind and may not be received well by the target audience. Any model used must be explored for relevance to the behavior that is being addressed (LaMorte 2016).

\subsubsection{Models/ Theories of BCC}

The Health Belief Model (HBM) is the most widely used theory in health education and promotion. This model was first developed in the 1950s by social psychologists working in the U.S. Public Health Services. The model was developed in response to the failure of a free tuberculosis (TB) health screening program. Since then, the HBM has been adapted to explore a variety of long and short-term health behaviors, including sexual risk behaviors and the transmission of HIV/ AIDS. The HBM holds that individual behavior depends on a number of beliefs about threats to an individual's wellbeing and the effectiveness and outcomes of particular actions or behaviors (University of Twente 2017)

Perceived threat is at the core of the HBM as it is linked to a person's 'readiness' to take action. The health belief model suggests that people's beliefs about health problems, perceived benefits of action and barriers to action, and self-efficacy 
explain engagement, or lack of it, in health-promoting behavior (University of Twente 2017). A stimulus, or cue to action, must also be present in order to trigger the health-promoting behavior. The HBM has the following constructs that are used to explain behavior.

The Perceived susceptibility is about a person's subjective perception of the risk of acquiring an illness or disease. There is wide variation in a person's feelings of personal vulnerability to an illness or disease. Perceived severity on the other hand alludes thata person's feelings on the seriousness of contracting an illness or disease or leaving the illness or disease untreated. There is wide variation in a person's feelings of severity. Often, a person considers the medical consequences, such as death or disability, and social consequences, including family life and social relationships, when evaluating the severity.

Perceived benefits are about individual's perception of the effectiveness of various actions available to reduce the threat of illness or disease. The course of action a person takes in preventing or curing illness or disease relies on consideration and evaluation of both perceived susceptibility and perceived benefit, such that the person would accept the recommended health action if it was perceived as beneficial (University of Twente, 2017).

Perceived barriers focus on a person's feelings on the obstacles to performing a recommended health action. The person weighs the effectiveness of the actions against the perceptions that it may be painful, time consuming expensive etc. Due to this the individual is likely to take action commonly known as cue to action. However, this needs stimulation to trigger the decision-making process to accept a recommended health action. These cues can be internal or external.

Self-efficacy is the level of a person's confidence in his or her ability to successfully perform a behavior. This construct was added to the model in mid-1980. Self-efficacy is a construct in many behavioral theories as it directly relates to whether a person performs the desired behavior.

The Integrated Behavioral Model (IBM) is a combination of two theories: The Theory of Reasoned Action (TRA) and the Theory of Planned Behavior (TPB). TRA asserts that behavioral intent is created or caused by two factors: the attitudes and our subjective norms. Meaning, the evaluation of behavior outcomes determines one's attitude about the behavior, and those normative beliefs. On the other hand, person's motivation to comply determines one's perception of the subjective norms about the behavior (SNV, 2016).

Together, attitude and subjective norms determine one's intention to perform the behavior, with intention being the most crucial determinant of behavior adoption. The TPB is a theory that links one's beliefs and behavior (SNV 2016). TPB uses the constructs of TRA, but adds an additional element of control as a determinant of behavior. In addition, the following factors may transcend intention, and directly affect whether or not the behavior is carried out. They include: knowledge and skills to perform the behavior, salience of the behavior, environmental constraints, and habit.

The Needs, Opportunity, Ability Model focuses on the environmental-level factors, which is relatively rare in social psychological models (GCN and COI 2009). The Needs, Opportunity, Ability (NOA) model of consumer behavior incorporates factors at the environmental level. At the individual level, intentions are formed through both 'motivation' (which is driven by needs and opportunities) and 'behavioral control' (agency) (which is driven by needs and opportunities)

The Theory of Planned Behavior looks at environmental factors within the context of 'perceived behavioral control. This theory provides an example of an intention-based model. According to the model, the key factors influencing behavioral intention are: attitudes towards the behavior, subjective norms and perceived behavioral control or agency (GCN and COI 2009).

This theory emphasizes the role of perceived behavioral control as an influence on behavioral intentions and actual behavior. Control beliefs are important determinants of the perception of behavioral control, and are extremely important for understanding motivation. The perceived behavioral control of a person who is uncertain of their ability to execute a behavior may be influenced by their perception of their personal resources, such as their own abilities, selfesteem and confidence, and the time and money that are required to be successful.

Perceived control can have a direct effect on behavior, bypassing behavioral intentions. For example, people may fail to stick to weight loss or exercise programs possibly because there is a doubt in their mind over their own behavioral control, and their ability to stick to the program and avoid temptation. Past behavior also plays a role in behavior change.

The Theory of Interpersonal Behavior builds on intention-based models by including external factors, notably habit as part of 'facilitating conditions. The more times a behavior is repeated, the more automatic and less deliberative it becomes (GCN and COI 2009).

The theory explains individuals' behavior in terms of what they have always done (habit), by what they think they should do (social norms) and by the consequences, they associate with a behavior (perceived consequences). The model also contains aspects that are directly related to the individual, for example attitudes, genetic factors, intention, and behavior and others that are related to the individual's environment, for example culture, facilitating conditions, and social situations (GCN and COI 2009).

\subsubsection{Communication Channels}

A channel is the way a message is disseminated. There are a wide variety of different communication channels ranging from basic face-to-face conversation, telecommunication channels like the telephone or e-mail, and computational channels like the medical record. It is important to know which channels can most effectively reach particular target populations. The following are some of the channels that can be used to disseminate health communication messages (Knowledge for Health 2017).

Mass media can be used for advocacy as well as raising awareness to the public about the general situation affecting the society. The message is designed to stimulate an appetite for learning and participation through regular 
dialogue with the affected community. This type of behavior change communication and social mobilization can work when actions, messages and materials are strategically planned, this way; the sender of the message is sure that the messages, materials and methods of dissemination, whether interpersonal, group or mediated, are socio-culturally acceptable (FHI and USAID 2002).

Communication through mass media can ensure that correct information reaches a specific population and can model positive attitudes. However, the aspect of policy becomes important at this point especially when an individual or community is motivated to attempt new behaviors.

Messages can be delivered through mass media, for example, television or radio spots; articles in periodicals; or material in brochures, posters, flip charts, picture codes or comics or in-person, by health workers, peer educators, counselors, or other trained personnel. Additional means of delivery include musical or dramatic performances and community events. Messages can be reinforced with "gimmicks" such as key chains or stickers. Each medium has its own advantages and disadvantages (FHI and USAID 2002).

Local networks also known as community networks and traditional media refers to media specific to a given city or region. This includes: workshops, street theatre, peer education and outreach rallies, public meetings, and local newspapers and radio stations. Advantages: Good for spreading new ideas through social networks and, over time, encouraging widespread support throughout the community (USAID 2010).

Materials, such as brochures, can be distributed as part of the activities. On the other hand, peer education is a cornerstone of all interventions with target populations. Peer education has an overwhelmingly positive impact on STI or HIV incidence and risk behaviors. Peer educators can help reach specific groups, model safe behaviors stimulate community discussions and provide referrals to appropriate services. Call centers, websites or outreach programs, have a significant impact on the way people experience change (USAID 2010).

Interpersonal group communication is another form through which the message can be disseminated to reach the target audience. Interpersonal channels are often face-to-face communication, such as counseling and telephone hotlines. Interpersonal form of communication can be more credible and specific because it takes place one-on-one with trusted sources. Interpersonal group communication is relevant if there is a national campaign. In some cases, the interpersonal messages are linked to messages in the mass media. Use health personnel such as Community Health Workers (CHVs) traditional birth attendants etc. to provide messages to the community at each contact (Knowledge for Health 2017).

\subsection{Health Communication Planning, Campaigns And Strategies}

Planning in Health communication is required because it permits preparation in advance and track successes and failures by ensuring that all opportunities are maximized. In order to achieve this, there are nine steps that should be followed (CDC 2018).

The first step entails formation of planning team and setting up of the budgeting rules. The selected team must be knowledgeable and influential and also must have a sense of the budget. While forming a team, the following questions should be considered. Who will be involved in the planning and budgeting process of the health communication campaign to optimize the organization's knowledge, resources, partners and decision-making process? Is there staff or volunteers in the participating organization that could be recruited in the team? What about from outside the organization?

How will you ensure that your planning process will include the input of representatives of the intended audience, as well as key partners \&informants? Are you working with a predetermined budget or will you establish the budget after you have identified what is needed to achieve specific objectives? All these questions are important (CDC 2018).

The second step is about determining of the unique focus and audience of the campaign. At this point you need to be clear on the nature and scope of the health issue that you are addressing. For instance, some health issues are very sensitive and you need to handle them with care not to cause more damage to already affected people. You are required to identify specific factors that the campaign will focus on, determine the key audience(s) and clearly articulate communication objectives for the campaign. To achieve this, some of the questions can used to guide individual developing the campaign. For example: what is the nature and scope of the health issue you want to address (describe \& quantify)? Which factors explaining the issue you want to focus on? Who specifically do you want to reach (key audience)? What do you want them to know, think and/ or do? (O'Sullivan, Yonkler, and Merritt, (2003).

The third step is about analyzing the audience and setting up the objectives. The focus at this point is on identification of aspects of the demography, behavioral and lifestyle profile of the audience that will be helpful in all aspects of planning. This involves the identification of current knowledge, perceptions, attitudes, motivations, as well as barriers to the adoption of behaviors.

Identify the most influential and supportive individuals and groups for instance community elders, religious leaders' political leaders etc. This will help develop testimonials, select messengers and partnerships. Identify the best channels to reach the audience. Optimize what you know and consider low-cost research to establish base line data and gather insight on the audience. Not all affected persons in the community will respond similarly to a given campaign and organization. Set realistic objectives based on the audience analysis (O'Sullivan, Yonkler, and Merritt, (2003).

It is important to identify opportunities and challenges as the fourth step. Here you may need to partner with other organizations/individual with the similar objectives. You are required to identify promotional and partnership opportunities that you should use. Identify your competition and other challenges to be considered in developing your strategy. You should consider targeted channels in order to avoid undesirable opposition or backlash. You may want to involve partner organizations and key community informants in the planning process to take their concerns and suggestions into account due to their influence in the society (National Collaborating Centre for methods and tools (NCCMT 2007). 
Selection of channels and partners is the fifth step that involves selecting various interpersonal and media channels, as well as any appropriate events based on the audience analysis, contextual considerations and budgets. Ensure that you have optimal frequency and timely exposure in each channel. Establish partnerships with organizations and people that will provide credibility for the messages and access to the target persons or community you want to reach (FHI and USAID 2002)

Develop and pre-test messages in the sixth step. This can be done by determining the content and type of appeal for messages. Also identify messengers and consistent branding elements for the campaign. A focused brief must be provided to message developers before pre-testing the messages and materials. Pre-testing is important because it helps to ensure that the message you plan to disseminate will be well understood (FHI and USAID 2002).

Monitoring and evaluation is an important step that should be addressed. This is commonly done before the actual implementation of the program. This is to ensure that it is included in the timetable and budget (FHI and USAID 2002). You need to map out how the campaign is expected to work. This is critical because it ensures that the right outcomes are measured. Here you need to develop the campaign's logic model, establish a systematic review of the implementation for example monthly, quarterly yearly depending with program. Ensure that activities are actually reaching the intended audience and evaluate progress toward the objectives. You can use baseline data and a methodology to evaluate progress. Establishing the timetable is the next step. This includes specifying the tasks, persons that will be involved and deadlines to ensure effective and efficient implementation. Make sure that you assign competent and dedicated person to oversee the initiative. Sometimes different tasks are assigned to all persons involved in the implementation of the campaign at this point. Tasks such as analyzing target audience, developing the message, establishing partnerships, pre-test, monitoring, evaluating results etc. are some of the activities that you need to include in you plan (NCCMT 2007).

Establishing of the budget is the final step in health communication planning, campaigns and strategies. It is important to provide realistic budget outlining expenses, sources of revenue and in-kind contributions. Your budget should include both expenses and revenues (O'Sullivan, Yonkler, and Merritt 2003). Revise your objectives and strategy based on confirmed revenues. Look for opportunities, leverage your personal contacts and develop strong cases when seeking additional funding.

\subsubsection{Health Communication Campaigns}

Campaigns seek to affect large audiences and bring substantial resources to the task (sometimes monetary, sometimes voluntary, sometimes through collaboration with other institutions). Campaigns attempt to influence adoption of recommended behaviors by influencing what people know and believe about the behavior, and/ or by influencing actual and/ or perceived social norms, and/ or by changing actual skills and confidence in skills (self-efficacy), all of which are assumed to influence behavior (NCCMT 2007).

A health communication campaign is an intervention that "intends to generate specific outcomes or effects, in a relatively large number of individuals, usually within a specified period of time, and through an organized set of communication activities. Public health campaigns are designed to influence a population to maintain or improve its health status. To accomplish this, campaign developers must understand the link between behavior and health status for the population of interest (NCCMT 2007).

Effective health communication campaigns use various methods to reach intended audiences. These includes: Media Literacy which teaches intended audiences to analyse media messages to identify the sponsor's motives; also teaches communicators how to create messages geared to the intended audience's point of view. Media Advocacy on the other hand is about influencing the mass media's through selection of topics and shaping the debate on these issues that seek to change the social and political environment in which decisions on health and health resources are made (CDC 2018)

Advertising entails placing paid or public service messages in the media or in public spaces to increase awareness of and support for behaviour change. Entertainment Education is an effective campaign method that seeks to include health-promoting messages and storylines into entertainment and news programs or to eliminate messages that counter health messages; can also include seeking entertainment industry support for a health issue (CDC 2018). Individual and Group Instruction also influences, counsels, and provides skills to support healthy behaviours and finally Partnership Development helps to increase support for a program or issue by harnessing the influence, credibility, and resources of profit, non-profit, or governmental organizations (CDEC 2018).

\subsubsection{Health Communication Strategies}

There are a variety of popular strategies for promoting healthful habits in a target audience. The upstream approach and downstream approach are commonly used. Upstream approach targets audiences, such as community leaders, government officials, and other influential individuals. These people are important because they have interpersonal influence, or can create change through their relationship with the target audience to influence health campaign. Also, they are more likely to have the ability to reform environmental factors that can shape behavior or influence policy and groups that work at the national or community level (NCCMT 2007).

Downstream approach on the other hand focuses mainly on two examples of which are social marketing and social norms campaigns. Many campaigns target the upstream audience first and then focus their attention on the downstream audience. However, the two approaches can be used interchangeably or exclusively (NCCMT 2007).

A campaign approach to health communication can help the health communicator design and disseminate effective messages consistently and strategically. Health communication campaigns can take many forms, address different objectives, and use a variety of media. They are usually designed: to influence people's beliefs and actions toward 
their health or the health of others, for specific target audiences or groups, and hardly for the entire population, for implementation within a particular span of time and to be integrated with various media and other communication efforts to educate an audience about a health-related topic.

A good strategy is critical to the success or failure of any health communication campaign because it provides the linkage between the how and why components. It provides a roadmap and sense of direction for generating the essential messages while also offering a rationale for the various actions that are proposed (NCCMT 2007).

\subsection{Message Development and Production}

Message is very important in health communication campaigns. This will prompt us to look at the following definition. Health communication is the process of promoting health by disseminating messages through mass media, interpersonal channels and events. The media is an important ally in any public health situation. It serves the role of being a source of correct information as well as an advocate for correct health behaviors. But before the media can take on that role, it needs to understand the virus, the issues surrounding it, policy and practices, and finally, recommended correct behaviors (NCCMT 2007).

Health promotion is the process of enabling people to increase control over, and to improve, their health. It moves beyond a focus on individual behavior towards a wide range of social and environmental interventions. Health education helps people to make wise choices about their health and the quality of life of their community (NCCMT 2007).

Communication helps to equip people with the facts, ideas and attitudes they need to make informed decisions about their health. Communication occurs when a message is transmitted and received. Message becomes important when it is heard, understood and believed so that is can pave the way for desired changes in behavior.

The following are steps in effective message development. A Good message is usually short, accurate and relevant. It must then be disseminated in the language of the target audience using vocabulary that is appropriate for that audience. The message tone can be humorous, didactic, authoritative, rational or emotionally appealing. It may be intended as a onetime appeal or as repetitive reinforcement. It is often necessary to develop several versions of a message depending on the audience to whom it is directed (UNESCO, UNICEF and WHO1997).

Messages that are meant to communicate health issues or effect health behavior change must clearly convey information that ensures public understanding and limits the chances of misunderstanding (USAID 2017). Such messages should be developed with the aim to increase knowledge, change attitudes and encouraging new behavior. Development of Messages directed for health behavior change should adhere to the following: message concept development which must consider the kind of message to be conveyed, and the messages should be simple, clear and seek to offer benefits and practical solutions that meet people's needs (USAID 2017).

When developing health communication message, it is important to work with health professionals. This will enhance the accuracy of the technical information. You can as well work with communication professional to help you produce high quality products. Follow the 7 'C's of effective communication, pretest and retest with the target audience revising to satisfy the target audience and produce messages (USAID 2017).

There are several dimensions of a message that should carefully be considered in order to meet goals and objectives. These are: appeal, approach, content must have meaning to the audience, text or images can be used, context and the use a source that the audience normally utilizes for information and one that the audience respect (Unite for Sight, 2009).

The aim of health communication message is to make positive impact towards behavior change. To achieve this, the message must be well delivered. Effective message delivery takes a process by allowing the exchange or passing of information by a sender to a receiver with meanings, thoughts and information being exchanged to produce mutual understanding and hopefully meaningful interaction (Unite for Sight (2009).

The process of transmitting this information from an individual to another (or group) is a very complex process with many sources of potential error. The meaning must be retained during the transmission of the message. There are six steps to help the sender of the message to be clearer when delivering the message. They include: purpose and message, channel of communication, delivering the message, potential barriers, check for understanding and feedback (Unite for Sight (2009).

As much it is important to deliver health messages effectively, there are challenges that may hinder this course. For example, failure to choose the most appropriate channels in delivering a message can cause more trouble. In health communication, if you use print media such as newspapers, and magazines you will only reach members of community that are literate and can read and write. Community radio become more appropriate since the message will be disseminated in the local language of the target group.

\subsection{Information, Education and Communication}

Information, education and Communication (IEC) is a public health approach aiming at changing or reinforcing health-related behaviors in a target audience, concerning a specific problem and within a pre-defined period of time, through communication methods and principles (WHO 2014). IEC is a pre-planned, concentrated educational endeavor with a specific objective focused towards specific program goals in order to reach an audience either in individual or group setting through skillful use of relevant methods and media to bring about change in knowledge, belief, attitude and behavior.

The information, education and communication are an important aspect in health communication especially behavior change. The following are some of the roles of IEC: informing people about health, illness, disability, and ways in which they can improve and protect their own health including more efficient use of the health care delivery system. IEC is 
used to stimulate community dialogue, remove myths, misconceptions, and taboos, reduce stigma and discrimination, create demand for information and services, help people cope with difficult situations, breaks sensitive news, motivate people to change, adopt, and maintain more healthful practices etc. (Sachdeva, et al. (2015).

Change of behavior and adoption of a new idea or message does not occur spontaneously. There are various stages through which the process evolves. They are as follows: receiving information, processing/ understanding the received information, changing the health behavior, and finally maintaining it. The audience becomes distinct at different stages depending with the characters they uphold thus the need to introduce repeated reminders and reinforcements.

The following are common audience characteristics. Pre-knowledgeable are the type of audience that are unaware of the problem or the risk. Knowledgeable are aware of the problem and knowledgeable about desired behavior. Approving these is the audience in favor of the desired behavior. Intending are those who intends to personally take desired action. Practicing are those who practices the desired behavior and Advocating are the individuals who practices the desired behavior and advocates it to others (Sachdeva et al. (2015).

As much as behavior change is a process, it may not be easy to some people thus the challenge to health communication. However, the following are stimuli that influence people to change behavior. Physical stimulus which entails fear of future pain, positive reinforcements, discomforts, and memory of past pain, rational stimulus covers wellbacked reasoning and sound knowledge, emotional stimulus on the other hand intensity's attitude, feeling of fear, love, or hope. Skills may be applied based on ability to use a new behavior and social structure involves pressure group/ social norms/ family and personal ((Sachdeva, S. et al. (2015).

\subsubsection{Edutainment}

Edutainment also known as Entertainment Education (E-E), enter-educate, or infotainment is the act of learning through a medium that simultaneously educates and entertains. Through entertainment, people are also educated bringing in the aspect of education. Entertainment education is the process of purposely designing and implementing a media message both to entertain and educate, in order to increase audience members' knowledge about an educational issue, create favorable attitudes, and change overt behaviour. (Onuekwe 2015).

The main purpose of entertainment education programming is to contribute to directed social change which is defined as the process by which an alteration occurs in the structure and function of a social system. This change can occur at the level of individual, community, or some other system. The two major role of edutainment are: attracting audiences that are hard to reach and stimulating individual and collective curiosity.

\section{Impediments to Behavior Change Communication (BCC)}

Communication interventions have been used to change behavior however there are challenges in trying to change peoples' behavior. They include: not all people that are exposed to health messages will understand them and therefore, accordingly change their behaviour, not all people who will understand the messages will agree with them and not all the people who agree with the messages will change accordingly. Indeed, only a small percentage of the primary audience exposed to a message will go ahead to practice the new behavior (Sullivan 2011).

Change of behaviour is apriority in health communication although the change may not be realised immediately, there is need to promote behaviour change in the affected community. The following approaches by (Laverack2017) can be applied. Dissemination involves initiating and intensifying awareness campaigns by health agencies which include providing information to those who are open to learning about the desired behavior. Education is about promoting learning, comprehension and acquisition of skills needed to adopt new behavior for those that are motivated to learn. Persuasion approach enhances acceptance of new health beliefs values and behaviors through rational arguments (Edgar, 2012)

Dialogue helps to promote mutual understanding and agreement through interpersonal and group discussions, shared experience and social network. Entertainment is also important because it promotes enjoyment, emotional, stimulation, and excitement by exposing the audience to messages through music, dance, comedy and drama. And finally, compliance is enhanced through positive or negative sanctions threats or incentives (Laverack, 2017).

Low health literacy, limited access to information, relatively poor research activity in developing countries, proliferation of low-quality healthcare information on the Internet; and the inability of health workers to communicate with patients are some of the barriers to effective health communication. These barriers can however, be addressed through persistent health literacy programs targeting individuals with marginal literacy skills, setting up of training centers that equip health communication professionals and health professionals with good communication skills (Laverack2017)

Focusing on the future of Behavior Change and Health Promotion, (Laverack2017) suggested the adoption of the following approaches. A behavior change approach, a strong policy framework to create supportive environment, and the empowerment of people to gain more control over making healthy decisions.

\section{Summary and Conclusion}

Behavior change cannot happen overnight due to interpersonal or environmental factors surrounding people. Behavior change is a process that must permit willingness of the affected person to change behavior. Physical stimuli rational stimuli, emotional stimuli, skills, family and personal networks and social structures are some of the factors that can prompt people to change their behavior.

Health communication strategies have been used to educate individuals or group to learn how to make right decision concerning illness affecting them through change of behavior. Behavioral models are used to help researchers 
understand behavior and also seek to explain why people behave the way they do. There are different social psychological models that seek to explain human behaviors.

The following are some of the models used in health communication. The Health Belief Model (HBM) is the most widely used theory in health education and promotion. The health belief model suggests that people's beliefs about health problems, perceived benefits of action and barriers to action, and self-efficacy explain engagement, or lack of it, in healthpromoting behavior.

The Integrated Behavioral Model (IBM) is a combination of two theories: The Theory of Reasoned Action (TRA) and the Theory of Planned Behavior (TPB). TRA asserts that behavioral intent is created or caused by two factors: the attitudes and the subjective norms. The attitude and the subjective norms determine one's intention to perform the behavior, with intention being the most crucial determinant of behavior adoption.

The Needs, Opportunity, Ability Model focuses on the environmental-level factors, which is relatively rare in social psychological models. Similarly, the Theory of Planned Behavior looks at environmental factors within the context of 'perceived behavioral control. This theory emphasizes the role of perceived behavioral control as an influence on behavioral intentions and actual behavior.

The Theory of Interpersonal Behavior builds on intention-based models by including external factors, notably habit as part of 'facilitating conditions. The theory explains individuals' behavior in terms of what they have always done, what they think they should do and the consequences they associate with a behavior.

As much as behavioral models are important they have limitations. Models are deliberately simplified to aid understanding, and 'do not account for all the complexities of behavior. Most models do not segment the target population however different factors are likely to carry different weight for different people.

Mass media message are disseminated through various channels to promote or raise awareness to the public to change behavior regarding health issues affecting them. However, it is important to ensure that correct information reaches a specific population and model positive change. Through education, people are informed about health problems and ways in which they can improve and protect their own health including more efficient use of the health care delivery system.

Health communication is a powerful and fundamental human interaction that can positively influence behavior change towards health problems affecting the society. The interaction at intrapersonal level, interpersonal level, through mass media, community engagement information and communication can increase knowledge, change attitudes and norms and cultural practices and produce changes regarding health problems.

Frequent health promotions are likely to cultivate the desired behavior change resulting to the prevention of diseases emanating from health-related behaviors. Behavior change models have been used in the development of strategies that foster protection, reduces risk behaviors, and encourage adoption and maintenance of positive behaviors. In addition, the behavior change models are used in the implementation, and evaluation of health-related behavior change interventions, however they have limitations.

Individual factors as well as societal factors contributing to behavior may require different approach to produce positive impact. Similarly, different intervention measures to behavior change can be applied to gain positive result.

The effort to bring about positive behavior change in the society should involve all stakeholders i.e. the government, the community, the health service providers, mass media and the Non-Governmental Organizations (NGOs). Sometimes people need frequent reminder and reinforcement which can be strategically delivered if all involved parties are well coordinate and willing to work towards a common goal which is positive change of behavior to prevent health problems.

\section{Reference}

i. Adler, R. \& Rodman, G. (2003). Understanding Human Communication. 8th ED. New York: Oxford University Press.

ii. Bensley, J.R. \&Brookins-Fisher, J. (2018). Community and Public Health Education Methods: A practical Guide $4^{\text {th }}$ ED. Burlington: Jones and Bartlett Learning.

iii. CDC (2018). Health Communication Strategies: Available at www.npin.cdc.gov. Accessed on $8^{\text {th }}$ May 2018

iv. Edberg, M. (2015). Essentials of Health Behavior: Social and Behavior Theory in Public Health. $2^{\text {nd }}$ ED Washington DC: Jones and Bartlett.

v. Edgar, T (2012). Communication and Behavior Change Challenges to Limiting the Development of Antibiotic Resistance. Available at www.www.ncbi.nlm.nih.gov.Accessed on 9th May 2018.

vi. FHI. \& USAID (2002). Behaviour Change Communication (BCC) for HIV/ AIDS a Strategic Framework. Available at www.hivpolicy.org.Accessed on $5^{\text {th }}$ April 2018.

vii. GCN \& COI (2009).Communication and Behavior Change. Available at www.behaviourworksaustralia.org.Accessed on $5^{\text {th }}$ April 2018.

viii. Heider, M. (2005). Global Public Health Communication: Challenges, Perspectives and Strategies. Toronto: Jones and Bartlett.

ix. Knowledge for Health (2017).Effective Health Communication Strategy. Available at www.k4health.org.Accessed on $5^{\text {th }}$ May 2018.

x. LaMorte, W.W. (2016). Behavior Change Models: The Health Belief Model. Available at www.sphweb.b ijirdmanagerumc.bu.edu. Accessed on $20^{\text {th }}$ April 2018.

xi. Laverack, G. (2017). The Challenge of Behavior Change Health Promotion. Esjberg: University of Southern Denmark Press.

xii. Martin,R.L. \&Di Matteo, R.M. (2014). The Oxford Handbook of Health Communication, Behavior Change, and Treatment Adherence. New York: Oxford University Press. 
xiii. Milgrom, K. (2015). The Role of Health Communications in Behavior Change. Available at www.apcoworldwide.com.Accessed on $5^{\text {th }}$ April 2018.

xiv. National Collaborating Centre for methods and tools (NCCMT). (2007). Developing health communication campaigns. Available at www.nccmt.ca.Accessed on $18^{\text {th }}$ April 2018.

xv. NCBI (2002).Assessing Health Communication Strategies for Diverse Populations. Health Communication Campaign Examplar. Available at www.ncbi.nlm.nih.gov.Accessed on 23 ${ }^{\text {rd }}$ April 2018.

xvi. Onuekwe, E.C. (2015). Entertainment-Education for Health Behavior Change: Issues and Perspectives in Africa. Fort St Victoria: Friesen Press.

xvii. O'Sullivan, G.A., Yonkler, J.A., Morgan, W., and Merritt, A.P.A (2003).Field Guide to Designing a Health Communication Strategy. Available at www.http:/ / ccp.jhu.edu. Accessed on 18th April 2018.

xviii. Parvanta, C. a Public Health Communication Planning Framework. Jones \& Bartlett. Learning. LLC

xix. Sachdeva,S. et al. (2015). Information, Education, and Communication (IEC): A revisit to Facilitate Change. www.medind.nic.in.Accessed on 8th May 2018.

xx. Scutch field, D.F. \& Keck, W. (2003). Principles of Public Health Practice 2nd ED. New York: Thomson Delmar Learning.

xxi. SNV (2016).Behaviour Change Communication Guidelines. Available at www.http:// www.snv.org. Accessed on 26th April 2018.

xxii. Sullivan, C.(2011). Health Behavior Change: Understanding Stages of Change. Vol 25.Issue 2.Available at www.tandfonline.com.Accessed on $28^{\text {th }}$ April 2018.

xxiii. The Henry J . Kaiser Family Foundation (2004). Entertainment Education and Health in the United States. Available at www.kff.org.Accessed on 2nd May 2018.

xxiv. Unite for Sight (2009). The Role of Media in Health Promotion. Available at www.uniteforsight.org.Accessed on $26^{\text {th }}$ April 2018.

xxv. University of Twente (2017). Health Belief Model: Explaining Health Behaviors. Available at www.utwente.nl.Accessed on 14th April 2018.

xxvi. UNESCO, UNICEF/ \& $\&$ WHO(1997). Facts for Life: Guide to Planning Health Promotion for AIDS Prevention and Control. Available at www.who.org.Accessed on 16 th April 2018.

xxvii. USAID. (2010). BEHAVIOR CHANGE COMMUNICATION (BCC): Learning Resource Package. Available at www.moph.gov.af.Accessed on $5^{\text {th }}$ April 2018.

xxviii. USAID (2017).How to Develop SBCC Creative Materials. Available at www.thehealthcompass.org.Accessed on $6^{\text {th }}$ April 2018.

xxix. WHO(2009). Why Health Communication is Important in Public Health. Vol 87 No 4 .Available at www.whi.int.Accessed on $23^{\text {rd }}$ April 2018.

xxx. WHO.(2014). Child health and development: Information, education and communication. Available at.www.emro.who.int. Accessed on $4^{\text {th }}$ May 2018. 